\title{
SECTORING AND RECOMBINATION IN ILLEGITIMATE DI-MON MATINGS OF SCHIZOPHYLLUM COMMUNE*
}

\author{
MOSHE SHALEV, ${ }^{1}$ JUDITH STAMBERG ${ }^{2}$ and GIORA SIMCHEN ${ }^{1}$ \\ 1 Laboratory of Genetics, The Hebrew University, Jerusalem, Israel \\ 2 Genetics Unit, 155 Herzl St., Tel-Aviv University, Tel-Aviv, Israel
}

Received 28.x.71

\section{INTRODUCTION}

Somatic recombination in the basidiomycetes is a complex process, not well understood either in terms of its mechanics or its genetics, although it has served as a tool in genetic experiments involving this group of fungi. (The term "somatic recombination" is generally used, somewhat inaccurately, to refer to the reassortment of chromosomes or linkage groups between somatic nuclei. We conform reluctantly to this usage, and make a distinction throughout this work between somatic recombination, as defined above, and "somatic intrachromosomal recombination", to designate the formation of new combinations of linked genes.) Basidiomycetes are distinguished by a unique phase in their life-cycle, a stable and persistent dikaryophase, during which two genetically unlike nuclei are present in each cell. In nature, the dikaryon is the dominant phase of the life-cycle. Somatic recombination occurs with a low frequency and can have selective value in producing genotypes compatible with other mycelia, because of the mating system in these organisms.

In Schizophyllum, Coprinus and related genera two unlinked incompatibility or mating factors, $A$ and $B$, control sexual morphogenesis, the process which begins with exchange of nuclei between two monokaryons and culminates in the formation of fruiting bodies, meiosis and production of haploid spores. Two monokaryons are fully compatible, however, only if they differ in both their $A$ and $B$ factor specificities. Monokaryons which possess the same $A$ factor but different $B$ factors are semi-compatible; nuclei are exchanged and migrate through the mycelium of the recipients, but in an uncontrolled manner, and fruiting bodies are not formed. Monokaryons with the same $B$ factor and different $A$ factors are also semi-compatible, but nuclear migration does not occur and the heterokaryon is generally restricted to the area of confrontation between the monokaryons (see Raper, 1966, for general review of the system). The $A$ and $B$ factors each consist of two linked loci, $\alpha$ and $\beta$. An allelic difference at either $\alpha$ or $\beta$ is sufficient to confer a different specificity on two factors.

Dikaryotisation of a monokaryon following its mating with a previously established dikaryon was first described by Buller (1931) and was later called "di-mon mating" (Papazian, 1950). This phenomenon is believed to occur very frequently in nature (Raper, 1966). If at least one of the nuclear types of the dikaryon is compatible with the monokaryon, e.g.

$$
(A 1 B 1+A 2 B 2) \times A 3 B 3 \text { or }(A 1 B 1+A 2 B 2) \times A 2 B 2
$$

* Requests for reprints should be addressed to the second author. 
the monokaryon is readily dikaryotised; this mating is termed a "legitimate di-mon mating". If, however, neither nuclear type in the dikaryon is compatible with that of the monokaryon, e.g. $(A 1 B 1+A 2 B 2) \times A 1 B 2$, then dikaryotic sectors within the monokaryon occur infrequently; the mating is termed an "jllegitimate di-mon mating".

Various explanations have been offered for the origin of these irregularly occurring dikaryotic sectors in illegitimate di-mon matings. These have included the following: (1) migration of the original dikaryotic genome through the monokaryon and its re-establishment at the periphery of the monokaryon (suggested by Buller, 1941; reported by Papazian, 1950, and Swiezynski, 1962). (2) Fusion of the dikaryotic nuclei to form a diploid which haploidises, giving a new type of nucleus with reassorted and possibly recombinant chromosomes from both of the original nuclei; this new nucleus can then dikaryotise the monokaryon (suggested by Buller, 1931; reported by Papazian, 1954, and Swiezynski, 1962). (3) Fusion of the monokaryotic nucleus with one of the nuclei of the dikaryon to form a diploid which haploidises to give a new type of nucleus; this new nucleus can then form a dikaryon with the second type of nucleus from the original dikaryon (reported by Swiezynski, 1962, 1963). (4) Exchange of chromosomes between nuclei during mitotic nuclear divisions in which mitotic spindles overlap (suggested by Quintanilha, 1939); one of the resulting new nuclei can then form a dikaryon with the monokaryon. (5) Formation of a triploid nucleus which then haploidises to produce new types of haploid nuclei (suggested by Brunswik, 1924, and Ellingboe and Raper, 1962a). (6) A "novel recombinational process" called "specific factor transfer" in which the $A$ factor from one of the dikaryotic nuclei and the $B$ factor from the other are inserted into the genome of the monokaryon (reported by Ellingboe and Raper, 1962a, and Ellingboe, 1963).

The way in which the haploidisation occurs is unclear. In Coprinus, the data suggest that, as in the parasexual cycle of ascomycetes, diploid nuclei break down through aneuploid stages to form new haploids (Swiezynski, 1963; Prud'homme, 1965). In Schizophyllum, however, diploidisation followed by "precocious meiosis" has been the favoured explanation because of the high frequency of recombination between linked loci (Raper, 1961; Ellingboe and Raper, 1962a; Parag, 1962; Ellingboe, 1964).

The work described here was undertaken to determine the origin of dikaryotic sectors in illegitimate di-mon matings, and to compare frequencies of somatic intrachromosomal recombination with those of meiotic recombination. In Schizophyllum the latter process is known to be controlled by a series of "fine-control" genes which regulate frequencies of recombination in specific, short regions of the genome (Stamberg, 1968; Simchen and Stamberg, 1969a). Di-mon matings provide a system whereby products of somatic recombination can be recovered; the frequency and type of intrachromosomal recombinants can be compared with those formed by the same dikaryons during regular meiotic divisions.

\section{MATERIALS AND METHODS}

All strains used in this work were synthesised by crosses among the strains in our collection in Jerusalem. The strain which served as monokaryon in 
all matings was M7, of genotype $A \alpha 1-\beta 6 B \alpha 1-\beta 1$ pab- ade5-* Two sibling strains, DI and D2, of genotype $A \alpha 1-\beta 6 B \alpha 3-\beta 2$ ade $5^{-}$nic $2^{-}$were obtained from a cross and served as alternate members of the dikaryon; the other component of the dikaryon was one of three siblings, EI, E2 and E3 of genotype $A \alpha 4-\beta 1 B \alpha 1-\beta 1$ comp2 $p a b^{-}$nic2-. Linkage relationships of the markers used are given in table 2 .

Crosses to obtain data on meiotic recombination were performed in the usual way, on complete medium (Raper and Miles, 1958; Stamberg, 1968). Fruiting occurred at $32^{\circ}$ C. Detection of recombinants was performed according to the scheme given by Stamberg (1968).

Illegitimate di-mon matings were performed as follows. On the first day of the experiment a small inoculum of strain M7 was placed near the rim of a series of standard petri plates containing a migration minimal medium (composition: 0.5 g. $\mathrm{MgSO}_{4}, 0.46$ g. $\mathrm{KH}_{2} \mathrm{PO}_{4}$, I g. $\mathrm{K}_{2} \mathrm{HPO}_{4}$, $2 \mathrm{~g}$. dextrose, $5 \mathrm{~g}$. asparagine and $20 \mathrm{~g}$. agar per litre of distilled water). At

TABLE 1

Di-mon mating tests used to identify the mating factors in each nucleus of new dikaryotic sectors

Tester strains*

$\begin{array}{lllllllllllll}\text { A } \alpha-\beta / & 1-6 / & 4-1 / & 1-6 / & 4-1 / & 4-1 / & 1-6 / & 4-1 / & 1-6 / & 1-1 / & 4-6 / & 1-1 / & 4-6 / \\ \mathrm{B} \alpha-\beta & 1-1 & 1-1 & 3-2 & 3-2 & 1-2 & 3-1 & 3-1 & 1-2 & 1-1 & 1-1 & 3-2 & 3-2 \\ \begin{array}{l}\text { Examples of new } \\ \quad\end{array} & & & & & & & & & & & & \\ \begin{array}{l}\text { dikaryotic sectors: } \\ \text { 4-1/1-1+1-6/3-2 }\end{array} & \mathrm{F} & + & + & \mathrm{F} & + & + & + & + & + & + & + & + \\ 4-1 / 3-2+1-6 / 1-1 & + & \mathrm{F} & \mathrm{F} & + & + & + & + & + & + & + & + & + \\ 4-1 / 1-2+1-6 / 3-2 & + & + & + & \mathrm{F} & + & + & + & \mathrm{F} & + & + & + & + \\ 4-1 / 3-2+4-6 / 1-1 & + & \mathrm{F} & + & + & + & + & + & + & + & + & + & \mathrm{F}\end{array}$

+ indicates a compatible reaction, i.e. the tester monokaryon is dikaryotised.

$F$ indicates a "flat" reaction, i.e. the tester monokaryon becomes a common- $A$ heterokaryon.

* Twelve testers are needed to identify completely all the types of sectors formed.

the same time, dikaryons were synthesised by placing small inocula of strain DI or D2 together with EI, E2 or E3 on plates containing complete medium. The dikaryons were made in replicates and incubated at $32^{\circ} \mathrm{C}$. for 72 hours. After this time a plug $1 \mathrm{~cm}$. in diameter was removed from the growing edge of each of the dikaryotic replicates and inverted on to the edge of the growing monokaryons. Each monokaryon in the series of replicates received an inoculum from a different plate in the series of dikaryotic replicates. The plates containing these di-mon matings were incubated at $32^{\circ} \mathrm{C}$. for 72 hours, then removed to an illuminated $24^{\circ} \mathrm{C}$. incubator for an additional 72 hours. The plates were then examined for the presence of dikaryotic sectors.

In order to identify the composition of a sector, a small plug was transferred to a fresh plate of complete medium. Determination of the mating factors was made by a second series of di-mon matings. The mating factors of each type of nucleus within the sector were identified by the use of 12 monokaryotic testers (table 1). Nutritional requirements were identified by inoculating small plugs of the sectors on to minimal medium (Raper and Miles, 1958) with the appropriate supplements. The presence or absence

\footnotetext{
* Abbreviations: $p a b^{-}=$requirement for para-amino benzoic acid; ade5- = requirement for adenine; $n i c 2-=$ requirement for nicotinic acid; comp $2=$ compact morphology of the colony.
} 
TABLE 2

Frequencies of newly derived sectors in three di-mon matings

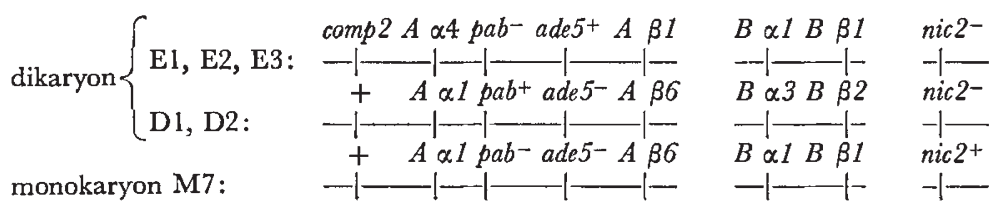

Frequency of sector type in sample

Type of sector recovered

$\overbrace{\begin{array}{ccc}\mathrm{D} 2+\mathrm{E} 1) & (\mathrm{D} 1+\mathrm{E} 2) & (\mathrm{D} 1+\mathrm{E} 3) \\ \times \mathrm{M} 7 & \times \mathrm{M} 7 & \times \mathrm{M} 7\end{array}}$

Nutritional $\overbrace{\%}^{*} \overbrace{\%}^{\times} \overbrace{\%}^{\text {origin (see }} \begin{gathered}\text { Type of } \\ \text { recombin- }\end{gathered}$ $A \alpha-\beta \mid B \alpha-\beta$ Morphology requirements* No. $\%$ No. $\%$ No. $\%$ Introduction) ation

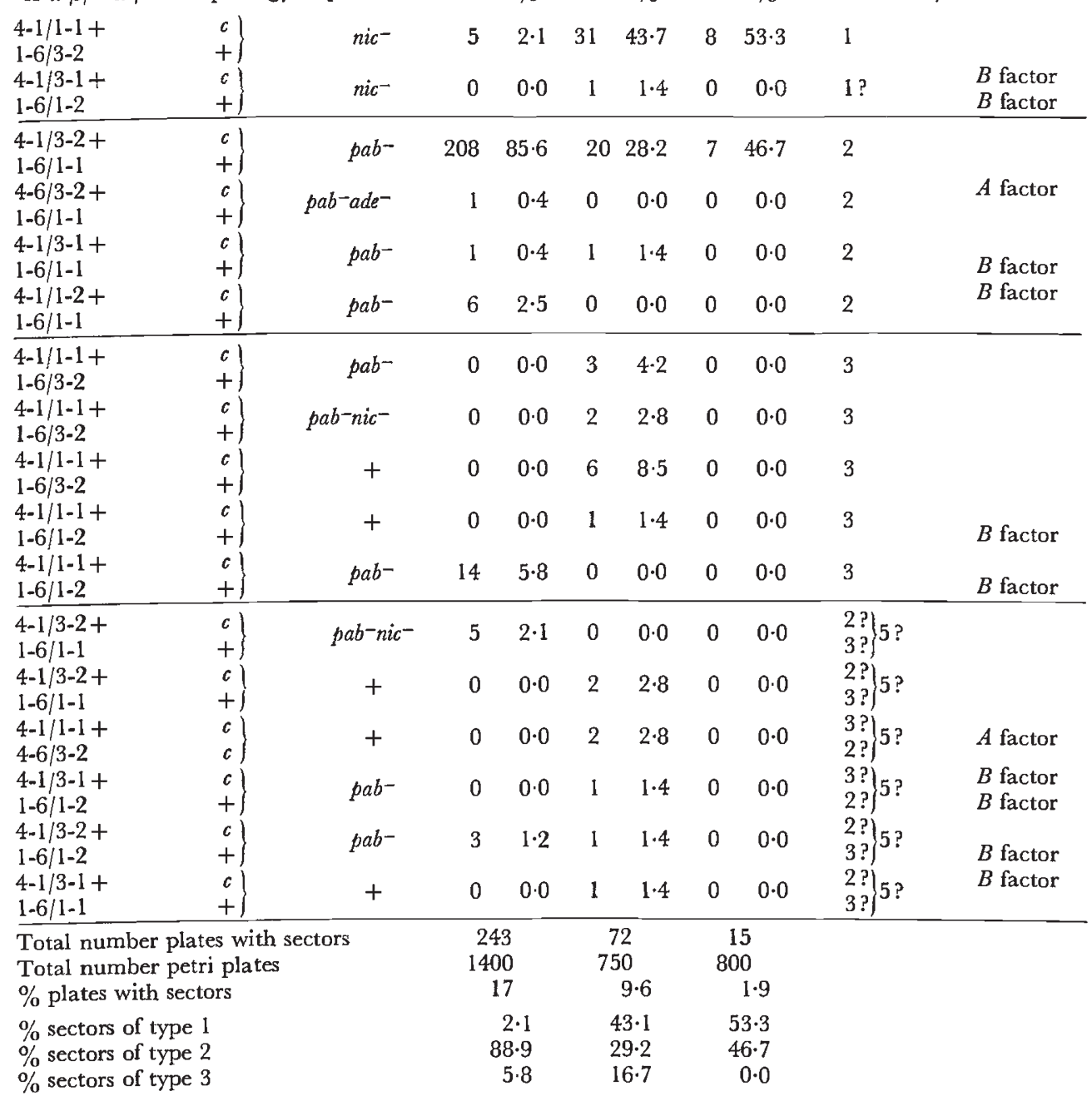

* The column "Nutritional Requirements" lists the phenotype of each sector. Because all the nutritional requirements are recessive, a sector displaying a nutritional requirement must be composed of nuclei all of which require the supplement. 
of the morphological marker comp2 in each type of nucleus within a sector was determined by allowing the sectors to fruit, collecting spores and classifying the spores with respect to their $A$ factor and morphology. If the majority of the compact spores carried $A \alpha 4$, for example, it could be concluded that the parental nucleus carrying $A \alpha 4$ also carried comp2, because the two markers are linked (Simchen and Stamberg, 1969b).

\section{(i) Meiotic}

\section{Results}

Frequencies of meiotic recombination were determined by analysing spores from the three dikaryons $\mathrm{D} 2 \times \mathrm{El}, \mathrm{Dl} \times \mathrm{E} 2$ and $\mathrm{D} 1 \times \mathrm{E} 3$ (table 3 ). In each cross both the $A$ and $B$ factors were identical in structure and specificity. No significant variation in frequency of $A$ or $B$ factor recombination among the crosses was observed (see $\chi^{2}$ values, table 3 ). The frequency of recombination between $A \alpha$ and comp 2 was significantly different in the second cross from that observed in the first and third crosses, a manifestation of the fine control of recombination in this region (Simchen and Stamberg, 1969b). Recombination within the $A$ factor was about three times more frequent than recombination within the $B$ factor.

\section{(ii) Somatic}

The frequency of sectoring was determined by counting the number of plates containing at least one sector. In the second and third di-mon matings no plates contained more than one sector. In the first mating, of the 243 plates with sectors, 219 had one sector, 21 had two sectors and 3 had three sectors. This distribution corresponds to expectations based on a Poisson distribution (expected values: 1148 plates without sectors, 218 with one sector, 21 with two sectors, and 13 with more than two sectors; $\chi_{(2)}^{2}=7.7$ $P \simeq 0.05)$. This indicates that when more than one sector appeared on a plate they were usually independent events. We took the more cautious interpretation, however, and scored multiple sectors as only one event. Thus our estimates of frequencies of sectoring in the first mating may be slight underestimates. The frequency with which sectors occurred was very significantly different in the three matings $\left(\chi^{2}>100, \mathrm{P}<0.001\right)$, ranging from about 2 to 17 per cent. (table 2), but the frequency of sectoring was repeatable for each mating. Each set of matings was made in duplicate; as there was no variation between duplicates the results were pooled.

The frequencies of the various types of sectors were also different in the three matings. In table 2 the types of sectors, their frequencies and their probable origin (see Introduction) are listed. Of the sectors produced in the first di-mon mating, 216 out of 243 (89 per cent.) consisted of the nucleus of the monokaryon plus a new nuclear type containing markers from both nuclei of the original dikaryon (type 2 sectors). The equivalent type of sector occurred in only 30 per cent. of the cases in the second mating, and in 47 per cent. of the cases in the third mating.

The frequency of type 3 sectors, in which a common- $A$ diploid haploidised to give a new nuclear type which then formed a dikaryon with the second nuclear type from the original dikaryon, also varies in the three di-mon matings (from 6 to 17 per cent.), as does the frequency of recovery of the original dikaryon as a new sector. The latter varies from 2 per cent. in the first mating to about 50 per cent. in the other two matings. 
196 MOSHE SHALEV, JUDITH STAMBERG AND GIORA SIMCHEN

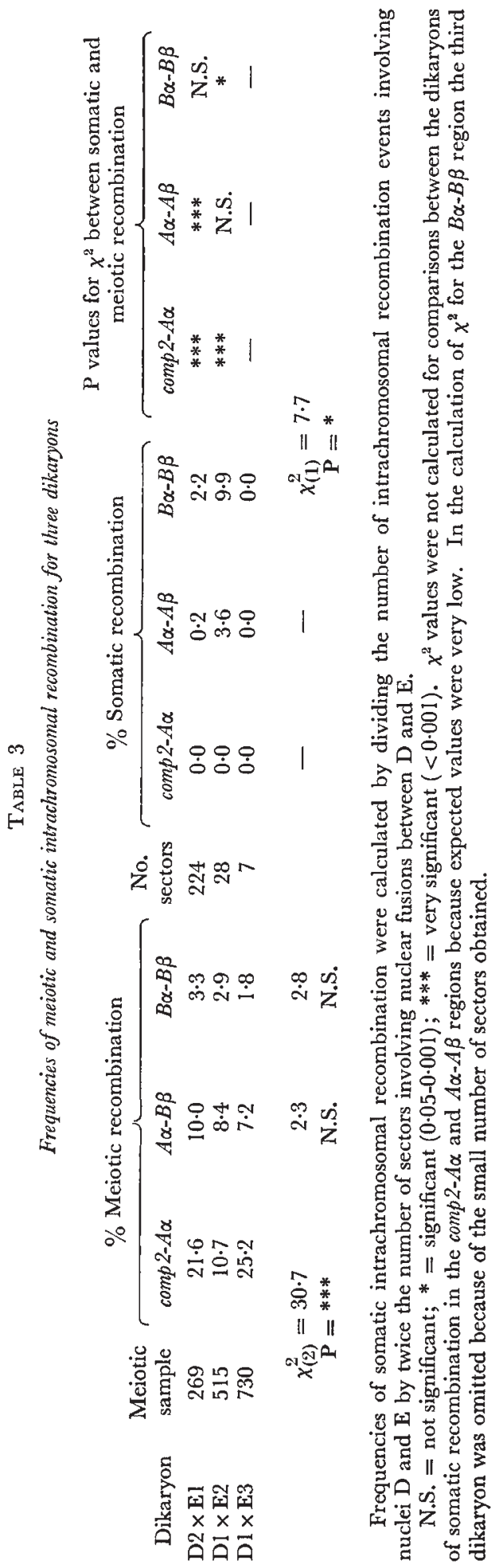


In addition, a small number of sectors was recovered, the origin of which cannot be explained in any simple way. These are grouped at the end of table 2. It is possible that they arose as haploid segregants from a triploid nucleus; alternatively, each nuclear type within the sector may have originated independently from different diploids, each of which haploidised to give new nuclear types. Thus, for example, the sector containing nuclei of types $A \alpha 4-\beta 1 B \alpha 3-\beta 2$ and $A \alpha 1-\beta 6 B \alpha 1-\beta 1$ and having the phenotype $p a b^{+} n i c^{+}$could have originated from a diploid formed by fusion of two nuclei of the original dikaryon, a haploid product of which subsequently formed a dikaryon with a haploid product of a common- $A$ diploid between a nucleus of the original dikaryon and a nucleus of the original monokaryon.

Frequencies of somatic intrachromosomal recombination are summarised in table 3. They were estimated by dividing the number of nuclear types recombinant for a given chromosomal region by twice the number of sectors containing nuclei with reassorted genomes, i.e. nuclei which gave evidence of having participated in a nuclear fusion event. Had all these nuclear fusions been between nuclei of types $\mathrm{D}$ and $\mathrm{E}$, then the calculation of somatic intrachromosomal frequency would be analogous to the calculation of the meiotic recombination frequency and the two could be compared. Since nuclei of the monokaryon M7 can also participate in nuclear fusions, however, the recombination events were subdivided into those between nuclei of types $\mathrm{D}$ and $\mathrm{E}$ (the nuclei of the original dikaryon) and those between types $\mathrm{D}$ and $\mathrm{M}$ (common- $A$ nuclei). The former are analogous to meiotic fusions between $\mathrm{D}$ and $\mathrm{E}$ and are given in table 3 . Only 25 per cent. of the products of reassortment were detectable, however, whereas 50 per cent. of the products of $A$ factor or $B$ factor recombination were detectable. That is, reassortment without crossing over should give nuclei of types $A \alpha 4-\beta 1$ $B$.1- $\beta 1, A \alpha 1-\beta 6 B \alpha 3-\beta 2, A \alpha 1-\beta 6 B \alpha 3-\beta 2$, and $A \alpha 4-\beta 1 B \alpha 3-\beta 2$ in equal frequencies, but only the fourth type would be recovered. Two of the four possible recombinant $A$ nuclear types would be recovered ( $A \alpha 4-\beta 6 B \alpha 3-\beta 2$ and $A \alpha 1-\beta 1 B \alpha 3-\beta 2$ ) whereas nuclei carrying a recombinant $A$ with $B \alpha 1-\beta 1$ would not. Therefore the calculation of somatic recombination frequencies requires a correction factor of 2 .

The number of sectors involving fusions between $\mathrm{D}$ and $\mathrm{E}$ was too small in the third mating for detection of any recombination. No recombination occurred in the comp2- $A \alpha$ region in any of the matings. Intra- $A$ and $-B$ factor recombination, however, occurred with a detectable frequency in the first and second matings. $A$ factor recombination in the second mating was at the level of the frequency of meiotic recombination; $B$ factor recombination occurred at the meiotic frequency in the first mating and at an even higher frequency in the second mating (see $\chi^{2}$ values, table 3 ).

\section{Discussion}

The results presented here show clearly that different illegitimate di-mon matings have very different probabilities of forming dikaryotic sectors. Sectors can originate in at least three ways. (1) Migration of the original dikaryon and its reestablishment as a new sector at the edge of the monokaryon, as previously demonstrated. Apparently this type of sector originates by migration of the two nuclei of the dikaryon without the formation of a diploid stage. If a diploid nucleus formed, migrated and subsequently 
haploidised, we should recover haploid nuclei containing reassorted $A$ and $B$ factors in equal frequency with haploid nuclei containing the original combinations of $A$ and $B$. That is, we should recover sectors of genotype $A \alpha 4-\beta 1 B \alpha 3-\beta 2+A \alpha 1-\beta 6 B \alpha 1-\beta 1$ and sectors of genotype $A \alpha 4-\beta 1 B \alpha 1-\beta 1$ $+A \alpha 1-\beta 6 B \alpha 3-\beta 2$ in equal frequencies. Since only the original combination is recovered, the original dikaryotic genome must migrate in the form of two haploid nuclei rather than as a diploid. (2) Fusion of two nuclei of the dikaryon and haploidisation to give a new nuclear type, compatible with the monokaryon, as reported previously. (3) Fusion of a nucleus of the monokaryon with a common- $A$ nucleus of the dikaryon and haploidisation to give a new nuclear type, compatible with the second nuclear type of the original dikaryon, as shown previously in Coprinus. These three types of sectors are formed in different frequencies in different di-mon matings. No evidence was found for "specific factor transfer", which would have produced sectors having the phenotype $p a b^{-} a d e^{-}$and forming spores none of which were of compact morphology. We consider the possibility of sectors forming by spindle overlap unlikely, because it is now known that in many fungi the nuclear membranes remain intact during mitosis (Bracker, 1967; Robinow and Caten, 1969). The possibility of rare triploid fusion cannot be ruled out, in the light of our anomolous sectors (the last group, table 2). On the other hand, it may be that once a rare diploidisation has occurred, a second has a greatly increased probability of occurring.

Why type 1 sectors should arise with different frequencies in the three matings is not clear. Since the monokaryon was the same throughout, the difference must lie in the dikaryons. Since the $A$ and $B$ factors were the same in all three dikaryons, some factor other than the incompatibility factors themselves must be responsible.

The formation of type 2 sectors must involve a fusion event between nuclei of the original dikaryon; thus, the difference in the frequency with which type 2 sectors occurred in the three matings could be due to different frequencies of diploid nuclei within the three original dikaryons, i.e. the dikaryon $\mathrm{D} 2+\mathrm{El}$ could have a high tendency to form diploid nuclei. That diploidy can occur in the mycelium of basidiomycetes is well documented. Stable common- $A$, common- $B$, common- $A B$, and fully compatible diploids have been recovered from Coprinus and Schizophyllum (Casselton, 1965; Parag and Nachman, 1966; Koltin and Raper, 1968; Mills and Ellingboe, 1969). A genetic basis for the existence of a stable diploid stage has been shown in one case (Koltin and Raper, 1968); therefore, it is reasonable to expect that the differences observed in the present study reflect genetic differences in frequency of diploid formation among the dikaryons. As the monokaryon of the di-mon mating was the same for all three matings, selection by the monokaryon could not account for the observed differences in frequency.

The recovery of type 3 sectors indicates that the monokaryon can participate in the formation of a new haploid nucleus; thus it cannot be assumed that the nucleus which does not participate in the diploidisation event is always that of the monokaryon. Type 3 sectors occurred in different frequencies in the three matings; since the monokaryotic component was the same for all, the difference can be attributed to the D nucleus. Type 3 sectors occurred with low frequency (about 6 per cent.) in the first mating, in which D2 participated; in the second mating, in which Dl was the nucleus 
with the same $A$ factor as that of the monokaryon, type 3 sectors occurred with a higher frequency (17 per cent.). (The sample size of the third mating was too small to test the prediction that, since $\mathrm{Dl}$ is the common- $A$ nucleus, type 3 sectors should again occur with about 17 per cent. frequency.)

The frequencies of somatic intrachromosomal recombination indicate that the levels of recombination within the incompatibility factors are as high or higher in somatic fusions than in meiosis. Recombination was not detected in the other region examined $(\operatorname{comp} 2-A \alpha)$, although at meiosis this region recombines more frequently than do the incompatibility factors. This finding is in contrast to the reports of Ellingboe and Raper (1962a) and Ellingboe (1964) that frequencies of somatic recombination in many regions throughout the genome were as high as the corresponding frequencies of meiotic recombination.

We recognise the possibility that nuclear selection could conceivably raise the apparent frequency of recombination in the incompatibility factors-i.e. that $B$ factor recombinants could be formed in very low frequency but be selected preferentially as the dikaryotising agent by the monokaryon-but we think it unlikely for two reasons. Firstly, in compatible di-mon matings, where the phenomenon of nuclear selection has been studied, selection is always for the $A$ or $B$ factor with maximal heterozygosity (Ellingboe and Raper, 1962b; Growe, 1963). That is, in a mating of the type

$$
(A \alpha 1-\beta 1 B \alpha 1-\beta 1+A \alpha 2-\beta 2 B \alpha 1-\beta 2) \times A \alpha 3-\beta 3 B \alpha 2-\beta 2
$$

the nucleus $A \alpha 1-\beta 1 B \alpha 1-\beta 1$ dikaryotises the monokaryon in the great majority of cases, even though the nucleus $A \alpha 2-\beta 2 B \alpha 1-\beta 2$ is equally compatible with the monokaryon. Thus, the nucleus of the monokaryon tends to select the $B$ factor with which it is doubly heterozygous. In our experiments, therefore, any selection by the monokaryotic nucleus ( $B$ factor $\alpha 1-\beta 1$ ) would have tended to give an excess of nuclei with the non-recombinant $B$ factor $\alpha 3-\beta 2$, rather than of nuclei with the recombinant $B \alpha 3-\beta 1$ or $B \alpha 1-\beta 2$, since both recombinant $B$ factors are heterozygous at only one locus with the monokaryotic nucleus whereas the non-recombinant $B$ factor is doubly heterozygous. Thus, if nuclear selection were operating it would tend to select reassorted but non-recombinant nuclei. If our estimate of $B$ factor recombination is distorted by nuclear selection, the true value must therefore be higher, not lower, than our estimate. Secondly, nuclear selection could not account for the difference in frequency of $B$ factor recombination between the two dikaryons, since the monokaryon was the same in both di-mon matings, and the $B$ factors in the two dikaryons were also the same.

That no somatic intrachromosomal recombination occurred in the comp $2-A \alpha$ region, though this region recombines with a high frequency at meiosis, indicates that there is no basis for postulating a " precocious meiosis" following diploidisation. Rather, we suggest that the high frequency of $A$ and $B$ factor somatic recombination reflects a fine control of somatic intrachromosomal recombination which selectively concentrates recombinational events within the incompatibility factors. The difference observed in the level of somatic recombination between the two dikaryons is also a manifestation of a specific fine control. Such a control could be of importance to an organism which lives in nature mainly as a heterokaryon. The ability to produce new incompatibility factor specificities by recombination would ensure successful confrontations between mycelia. 


\section{SUMmary}

1. Three illegitimate di-mon matings, involving a common monokaryon and identical incompatibility factors in the dikaryons, were performed in replicate series in Schizophyllum commune.

2. Dikaryotic sectors originated in at least three ways:

(i) Migration of haploid nuclei from the original dikaryon to a new site at the edge of the monokaryon.

(ii) Fusion of two nuclei of the original dikaryon followed by haploidisation to give a new nuclear type which formed a dikaryon with a nucleus of the monokaryon.

(iii) Fusion of a nucleus of the monokaryon with a nucleus, containing the same $A$ factor, from the dikaryon followed by haploidisation to give a new nuclear type which formed a dikaryon with the second type of nucleus of the original dikaryon.

3. A small number of sectors resulted either from triploid fusion or from multiple diploidisation events.

4. The total frequency of sectoring, as well as the relative frequencies of the three types of sectors, varied from one mating to another. These differences in frequency are attributed to some factor in the original dikaryons other than the incompatibility factors. others.

5. No evidence was found for "specific factor transfer" as reported by

6. Frequencies of somatic intrachromosomal recombination were as high as frequencies of meiotic recombination in the incompatibility factors. In an adjacent region, with a high meiotic recombination frequency, no somatic recombination occurred.

7. It is suggested that a fine control of somatic intrachromosomal recombination selectively concentrates recombinational events within the incompatibility factors.

Acknowledgments.-We thank Dr Y. Parag for generously defraying a part of our research expenses through his U.S. Dept. of Agriculture grant A10-CR-66. Thanks are also due to Dr Y. Koltin for valuable comments on the manuscript, and to Miss Kalomira Halevi for expert and devoted technical assistance.

\section{ReFERENCES}

Bracker, C. E. 1967. Ultrastructure of fungi. Ann. Rev. Phytopath., 5, 343-374.

BRUNSwIK, H. 1924. Untersuchungen uber die Geschlechts-und Kernverhaltnisse bei der Hymenomyzetengattung, Coprinus. Bot. Abh. K. Goebel, 5, 1-152.

BULler, A. H. R. 193I. Researches on Fungi, vol. IV. Longmans, Green, and Co., London. BULLF. A. H. R. 1941. The diploid cell and the diploidisation process in plants and animals, with special reference to the higher fungi. Bot. Rev., 7. 335-431.

CAsselton, L. A. 1965. The production and behaviour of diploids of Coprinus lagopus. Genet. Res., Camb., 6, 190-208.

GROWE, L. K. 1963. Competition between compatible nuclei in the establishment of a dikaryon in Schizophyllum commune. Heredity, 18, 525-533.

ELLINGBOE, A. H. 1963. Illegitimacy and specific factor transfer in Schizophyllum commune. Proc. Nat. Acad. Sci. (U.S.), 49, 286-292.

ELIINGBOE, A. H. 1964. Somatic recombination in dikaryon K of Schizophyllum commune. Genetics, 49, 247-251. 
ELLINGBOE, A. H., AND RAPER, J. R. 1962a. Somatic recombination in Schizophyllum commune. Genetics, 47, 85-98.

ELlingBoE, A. H., AND RAPER, J. R. 1962b. The Buller phenomenon in Schizophyllum commune: nuclear selection in fully compatible dikaryotic-homokaryotic matings. Amer. 7. Bot., 49, 454-459.

KOLTIN, Y., AND RAPER, J. R. 1968. Dikaryosis: genetic determination in Schizophyllum. Science, 160, 85-86.

mills, D. I., AND ellingboe, A. H. 1969. A common-AB diploid of Schizophyllum commune. Genetics, 62, 271-279.

PAPAZIAN, H. P. 1950. Physiology of the incompatibility factors in Schizophyllum commune. Bot. Gaz., 112, 143-163.

PAPAZIAN, H. P. 1954. Exchange of incompatibility factors between the nuclei of a dikaryon. Science, 119, 691-693.

PARAG, Y. 1962. Studies on somatic recombination in dikaryons of Schizophyllum commune. Heredity, 17, 305-318.

PARAG, Y., AND NACHMAN, B. 1966. Diploidy in the tetrapolar heterothallic basidiomycete Schizophyllum commune. Heredity, 21, 151-154.

PRUD'HOMмE, N. 1965. Somatic recombination in the basidiomycete Coprinus radiatus. In Incompatibility in Fungi, pp. 48.52 (K. Esser and J. R. Raper, eds.). Springer-Verlag, Berlin.

Quintanilha, A. 1939. Étude génétique du phénomène de Buller. Bol. Soc. Broter., 13, 425-486.

RAPER, J. R. 1961. Parasexual phenomena in Basidiomycetes. In Recent Advances in Botany, pp. 379-383. Univ. of Toronto Press, Toronto.

RAPER, J. R. 1966. Genetics of Sexuality in Higher Fungi. Ronald Press, N.Y.

RAPER, J. R., AND MILES, P. G. 1958. The genetics of Schizophyllum commune. Genetics, 43, 530-546.

Robinow, C. F., AND CATEn, C. E. 1969. Mitosis in Aspergillus nidulans. 7. Cell Sci., 5, 403431.

simchen, G., AND Stamberg, J. 1969a. Fine and coarse controls of genetic recombination. Nature, 222, 329-332.

SIMGhen, G., AND STAMBERG, J. 1969b. Genetic control of recombination in Schizophyllum commune: Specific and independent regulation of adjacent and non-adjacent chromosomal regions. Heredity, 24, 369-381.

STAMBERG, J. 1968. Two independent gene systems controlling recombination in Schizophyllum commune. Molec. Gen. Genet., 102, 221-228.

SWIEZYNSKI, K. M. 1962. Analysis of an incompatible di-mon mating in Coprinus lagopus. Acta Soc. Bot. Polon., 31, 169-184.

SWIEZYNSKI, K. M. 1963. Somatic recombination of two linkage groups in Coprinus lagopus. Genet. Polon., 4, 21-36. 